\title{
Damage Control Vascular Surgery in the Austere Environment
}

\author{
Shaun M. Gifford ${ }^{1}$ - Zachary M. Arthurs ${ }^{1}$
}

Published online: 4 February 2016

(C) Springer International Publishing AG (outside the USA) 2016

\begin{abstract}
Managing vascular trauma in an austere environment with limited resources will challenge even the most seasoned surgeon. Successful management requires immediate hemorrhage control, and then, the surgeon must make critical decisions for limb salvage to be successful. This chapter highlights the principles of vascular management and provides damage control techniques that can be applied in a wide array of trauma settings.
\end{abstract}

Keywords Vascular $\cdot$ Injuries $\cdot$ Shunts $\cdot$ Damage control surgery $\cdot$ Ischemia $\cdot$ Limb $\cdot$ Trauma

\section{Introduction}

The treatment of vascular injury in an austere environment presents unique challenges to successful management. Whether it is on the battlefield, in third world nations, or in the rural setting, the injured blood vessel demands quick identification, rapid control of hemorrhage, and expeditious repair to limit continued injury from ischemia. Damage control surgery, which has evolved over recent years, focuses on contamination and hemorrhage control, physiologic resuscitation, followed by definitive repair $[1,2]$. Repair is delayed until a time in which the

This article is part of the Topical Collection on The Military Perspective

Zachary M. Arthurs

arthursz@mac.com

1 Vascular Surgery Faculty, San Antonio Military Medical Center, 3551 Roger Brooke Dr., Fort Sam Houston, San Antonio, TX 78234 , USA patient is resuscitated and can tolerate extended operative times and where repairs are at a lower risk of failing. Mortality benefit in this approach when used appropriately is clearly established $[3,4]$.

In the setting of the disrupted blood vessel, distal ischemia is a unique factor that has driven the creation of additional management strategies. Vascular injury often leads to a potential state of physiologic disarray, but in the austere environment the required expertise in repair methods may be limited, or access to the appropriate equipment or supplies may not be available. Transport to facilities with these capabilities requires temporizing means to prevent further injury. For all of these reasons, strategies to temporize repair to allow for transport or delay in repair are important.

Damage control vascular surgery has evolved over recent years largely in response to the wars in Iraq and Afghanistan. Twelve years of war with the highest percentage of vascular injury in history has provided us the opportunity to evaluate prior management strategies and to evaluate new techniques [5•]. Due to advances in body armor and expedited transport, more individuals are surviving injuries that in prior wars would have lead to death. Strategies established in World War 2 and streamlined during the Korean and Vietnam Wars have provided a framework by which to create more modern approaches, and a yardstick by which to measure success. The large numbers of vascular injures, now totaling nearly 1600 injuries, have provided the ability to fine tune and to scientifically evaluate these new strategies [5•].

This review will focus on the current strategies of early identification and hemorrhage control, early re-establishment of flow and adjuncts that help to limit the loss of functional recovery that we employ today, not only on the battlefield, but also in civilian trauma centers. 


\section{Injury Identification and Hemorrhage Control}

The integral part of successful vascular trauma management is early injury identification. The classic "hard signs" of injury, which include active hemorrhage, expanding or pulsatile hematoma, thrill or bruit, or the absence of distal perfusion makes identification of the injury and the decision to intervene straightforward. In those without these findings, a high index of suspicion is needed based upon the injury pattern and mechanism of injury.

The most important tool to evaluate possible axial flow disruption is the continuous wave Doppler. This instrument is a low cost device ( $\$ 150$ approximately) that is necessary for personal deploying to or staffing austere locations. It is more reliable than palpation of pulses and offers an objective gage of blood flow that can be followed over time. Signal presence, quality, and comparison to the contralateral limb can identify potential injury. Closely related is the use of the ankle brachial index (ABI) or injured extremity index (IEI), which compares the pressure differential between an injured limb and the uninjured brachial pressure. This is calculated by finding the ratio of occlusion pressure below the level of suspected injury in the injured limb to that of the normal brachial pressure. A ratio of 0.9 or less indicates injury in $94 \%$ of cases and should prompt further imaging or surgical exploration [6].

It is important to note that physiologic derangement can impact the Doppler signal, IEI, or even the presence of a signal. Significant arterial spasm can occur, leading to suspicion of injury in the absence of a true vascular lesion. Ongoing resuscitation and patient warming can lead to improved distal perfusion. Continued evaluation with serial examinations during the warming and resuscitation period is needed to identify improvement in signals or IEI. Fox and colleagues evaluated the use of newly established resuscitation strategies using a 1:1 ratio of red blood cells and fresh frozen plasma in addition to platelet transfusion and cryoprecipitate in those combat casualties with vascular injury. He found that correction to normal physiologic parameters were improved with an aggressive resuscitation strategy and did not lead to worse limb salvage $[7 \cdot]$.

Often the initial identification of vascular injury and active hemorrhage occurs far from the operating room. On the battlefield or in the rural setting, buddy care and emergency medical technicians or combat medics are the first to potentially intervene. With active exsanguination from extremity wounds, manual pressure is helpful, but not always possible for transport to higher levels of care. Tourniquets have been instrumental in controlling extremity hemorrhage and reducing preventable mortality and morbidity over the past 12 years. Concerns regarding improper tourniquet utilization have been negated with proper first responder training. Currently, all active duty soldiers carry a tourniquet as part of the daily uniform. In addition, all soldiers are trained on tourniquet placement and hemorrhage control. Reports from Iraq and Afghanistan have shown a significant benefit and lack of adverse outcomes from tourniquet use (Fig. 1). This has led to the rapid adoption of tourniquets by many civilian trauma centers or systems (Figs. 2 and 3).

At a combat support hospital in Iraq, Kragh and colleagues prospectively examined early tourniquet outcomes over a 7month period [8]. Tourniquets were directly associated with improved survival rates. This represented early findings that tourniquets could be deployed with soldiers, soldiers could be trained to place them appropriately, and these efforts resulted in lives saved [8]. In a separate report, Kragh evaluated the risk of tourniquet use on increased morbidity and limb threat, a common misconception surrounding tourniquet use. Of 428 tourniquets applied to 309 injured limbs, only ten had nerve palsies and no amputations were due to tourniquet-related complications; this provided additional data to support tourniquet utilization and its safety [9]. Additionally, Beekley et al. found that the use of tourniquets in forward setting is directly associated with improved hemorrhage control in severely wounded patients with multi-system trauma and high injury severity scores [10]. Thus, tourniquet acceptance and widespread adoption represents one of the most dramatic point of care improvement in the last decade. The education and training implemented to ensure this technology was utilized appropriately has resulted in improved early hemorrhage control, improved limb salvage rates, and significantly lower mortality rates from extremity injuries and amputations.

However, approximately two-thirds of deaths among trauma patients related to hemorrhage are in anatomic areas that are not amenable to tourniquet application. This includes "junctional" injuries, typically located in the groin, axilla, or neck, and non-compressible intra-cavitary or torso injuries. Non-compressible torso hemorrhage continues to be the leading cause of death in injured soldiers. Nearly $13 \%$ of battlerelated injuries occur in this region with $17 \%$ comprised of non-compressible hemorrhage [11]. In those individuals with profound hypotension and injury patterns consistent with

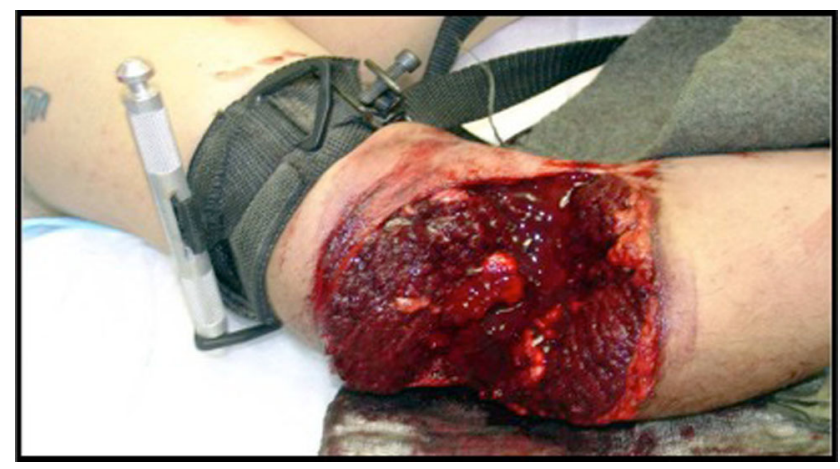

Fig. 1 Upper extremity tourniquet applied proximal to the antecubital fossa. Appropriate application leads to cessation of hemorrhage even in the setting of devastating injury 


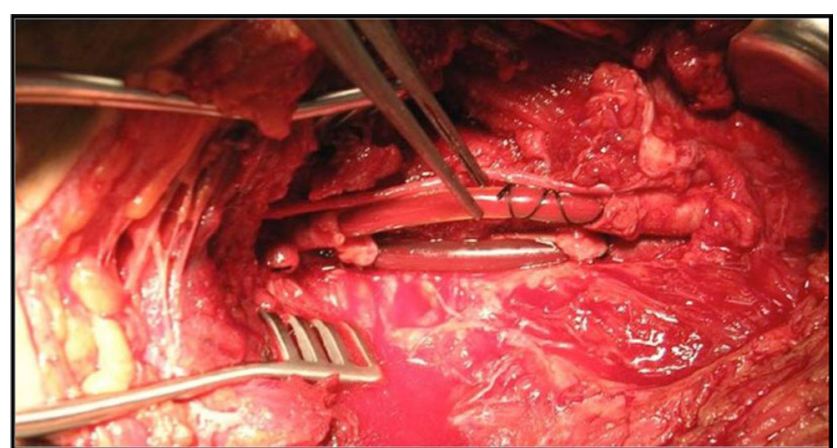

Fig. 2 Temporary vascular shunt (Argyle and Javid shunt) placed in the superficial femoral artery and vein secured with silk suture. Flow was preserved during transport to Echelon 3 facility where saphenous vein interposition could be performed

central vascular injury, the advent of bedside intravascular balloon occlusion devices provides the potential to obtain axial flow occlusion at the aortic level, so that resuscitation can catch up and the patient be transported to the operating room for exploration and repair. Historically, emergency thoracotomy provided this opportunity. The technique of resuscitative endovascular balloon occlusion of the aorta (REBOA) has gained major interest in recent years, with some data suggesting an improved overall survival and a lower rate of early death in several recent small trials [12]. A national multicenter prospective REBOA trial is ongoing and actively collecting data in civilian trauma centers.

\section{Re-establishment of Flow and Distal Perfusion}

Once the identification of vascular injury is made and operative intervention decided, the surgeon is faced with a decision on optimal injury management. Simple ligation, primary repair, or replacement with prosthetic or vein interposition are the most described options. Patient physiology and concomitant injuries, casualty tempo, ability to perform repair with supplies at hand, and potential for evacuation to a higher level of care all become part of the decision. Delay in limb

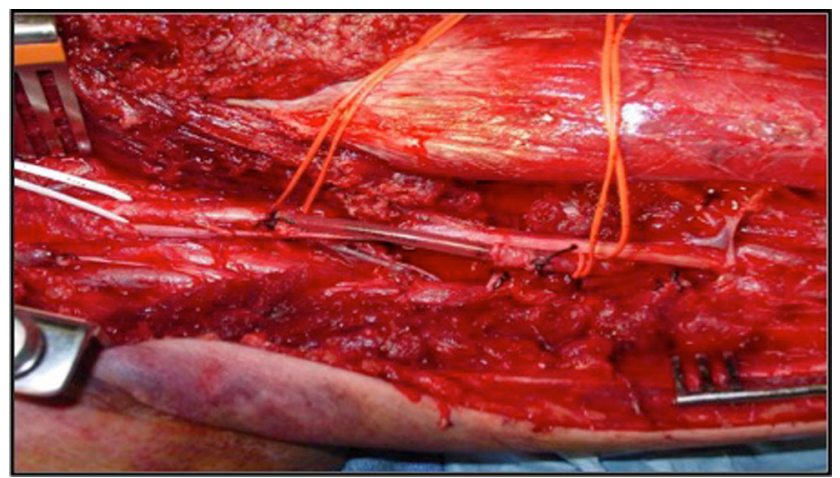

Fig. 3 Temporary vascular shunt (Argyle) placed in the brachial artery. Repair was successful with saphenous vein graft reperfusion clearly leads to worse outcomes and functional recovery, and damage control techniques should prioritize control of the bleeding and then restoration of at least some degree of distal perfusion. Recent reports from animal studies have demonstrated that irreversible damage and loss of functional recovery can occur as early as $3 \mathrm{~h}$ after ischemia begins $[13 \cdot, 14]$.

Although formal vascular repair is the gold standard for managing the injury and restoring flow, this is often time consuming and requires specialized skills and supplies. In order to quickly and more simply reestablish flow, temporary vascular shunts are necessary in austere locations and have been widely utilized by forward units over the past decade-plus of combat operations in Iraq and Afghanistan. Early vascular exploration identifies the injury location and provides hemorrhage control. If the artery thrombosed, thrombectomy and local heparin instillation prepares the inflow artery and outflow vessel. In mass casualty events, time cannot be taken for formal vascular reconstruction, nor can all trauma surgeons perform intricate reconstructions. Intravascular shunt placement is quick, requires no specialized equipment or supplies other than the shunt itself, and can be performed by most surgeons with limited vascular experience. If definitive repair is deferred to higher level of care, minimal debridement of the artery should be performed unless absolutely necessary for shunt placement. The shunt is inserted first into the distal or outflow vessel and then into the proximal or inflow vessel. These shunts should always be well secured with sutures, ties, or other methods so as to avoid displacement during transport. The most common shunts utilized are the Javid ${ }^{\mathrm{TM}}$, Argyle ${ }^{\mathrm{TM}}$, or Sundt ${ }^{\mathrm{TM}}$ shunts, although any appropriately sized hollow plastic tubing will suffice. It is our preference to choose a length of shunt that is redundant enough to allow a loop to be sutured to the skin. This allows the transport team and receiving surgeons easy access to Doppler the shunt as well as the outflow vessels.

With the shunt in position, patients can then transition to the next level of care or to the ICU for continued resuscitation with intact distal perfusion. Typically, these injuries are associated with segmental loss of the injured vessel, and demand formal vein interposition graft for revascularization. If the shunt occluded in route or if there is thrombus around the ends of the shunt at time of removal, thrombectomy should be performed. In addition, the artery requires debridement until uninjured intima is identified. After these steps are performed, there is often a gap that requires vein or prosthetic conduit to bridge the injury.

We now have several reports demonstrating excellent shunt patency after placement at forward locations in Iraq and Afghanistan [15-19]. Minimal complications occurred with no dislodgment reported (Table 1). Although thrombosis did occur in $4-22 \%$ of placements, shunt presence did not alter forma vascular. Once resuscitated and transported to a tertiary facility, the majority of injuries were reconstructed with 
Table 1 Temporary vascular shunts were described in several reports with a high patency rate and minimal complications. Limb salvage was excellent in the immediate postoperative period during evacuation stateside

\begin{tabular}{lllll}
\hline Report & Shunts utilized & Patency & Complications & Early limb salvage \\
\hline Chambers et al. 2006 & 27 & $78 \%$ & $22 \%$ shunt thrombosis & $85 \%$ \\
Rasmussen et al. 2006 & 30 & $86 \%$ & $14 \%$ shunt thrombosis & $92 \%$ \\
Clouse et al. 2007 & 43 & $86 \%$ & $14 \%$ shunt thrombosis & $93 \%$ \\
Taller et al. 2008 & 23 & $96 \%$ & $4 \%$ shunt thrombosis & $100 \%$ \\
Woodward et al. 2008 & 43 & $93 \%$ & $7 \%$ shunt thrombosis & $93 \%$ \\
\hline
\end{tabular}

autologous vein. Autologous vein, when available, was the conduit of choice, as complications of prosthetic conduit were high, with nearly $80 \%$ needing replacement due to early thrombosis or infection $[20,21]$. However, in the damage control setting, a repair with prosthetic interposition graft may be utilized as a temporizing measure instead of a shunt, with planned later prosthetic removal and replacement with a vein conduit. In a case control analysis comparing matched groups of extremity vascular injury with and without the use of temporary shunts, no detrimental effects of shunt utilization were identified, and in those with more severe injury, shunt placement improved limb and survival outcomes [22•].

In the austere setting, the majority of vein injuries can be managed with ligation or lateral venorraphy. Surgeons have attempted to utilize shunts for the vein with varying results. Shunts commercially available are extremely small (3-7 mm) compared to the veins in the pelvis and extremities. In addition, there is very little advantage to maintaining venous patency in the damage control setting. If the vein demands repair, this can be performed at a later time. While most veins tolerate ligation without consequence, the common femoral and the below knee popliteal vein are important points that drain parallel networks of the leg. Depending on the degree of venous collateralization in place prior to injury, ligation may be tolerated, but in some, it may result in severe venous hypertension and outflow obstruction. In the setting of combined arterial and venous injuries, severe venous hypertension can increase vascular resistance leading to early arterial repair thrombosis. For these reasons, venous repair should be considered when approaching combined arterial and venous injury through the common femoral artery/ vein and the below knee popliteal artery/ vein.

\section{Adjuncts to Limit Functional Loss}

We have discussed above the need to reestablish blood flow early in an effort to limit ischemia and maintain a viable limb. One consideration that must be evaluated is the impact of reperfusion injury and the potential for developing compartment syndrome and additional injury leading to poor functional outcome even in the setting of adequate distal perfusion.
In the civilian environment, surgeons have the luxury of serial exams, often performed by the same provider or trauma team. Clinical changes will be recognized and selective compartment decompression with fasciotomy performed. In the austere environment with high casualty volume and rapid evacuation to higher levels of care, routine serial examination and close monitoring is often limited or not possible. Prophylactic fasciotomy, when performed correctly, alleviates the risk of worsening injury or even limb loss due to development of compartment syndrome. Delay in transport, shunt thrombosis, or mass casualty events have the potential to limit the opportunity to decompress when compartment syndrome presents. For these reasons, liberal use of prophylactic, decompressive fasciotomy is advocated during the initial exploration and re-establishment of flow for most extremity vascular injuries.

Ritenour and colleagues evaluated over 640 fasciotomies performed in soldiers evacuated to Germany after injury on the battlefield. They found that when fasciotomy revision was required for incomplete decompression, additional muscle excision was required leading to worse limb function and higher mortality [23]. This underscores the importance of an early, complete decompression at the outset. It is rare to perform upper arm or thigh fasciotomies; however, all austere or military surgeons should be well versed with lower limb (calf) and forearm fasciotomies. Hand and feet fasciotomies are controversial, and from our perspective have little role for the austere surgeon. If fasciotomies are indicated, a full and complete release of all compartments should be performed, and limited or "mini" fasciotomies should be avoided. For the most commonly affected area, the calf, this means a twoincision (lateral and medial) fasciotomy with complete release of all four compartments.

\section{Tourniquet Application and Vessel Ligation as Temporizing Maneuvers}

Our vascular department has had the privilege of teaching the Department of Defense (DOD) Emergency War Surgery Course, the DOD Combat Extremity Surgery Course, and American Association of Orthopedic Surgery Extremity Course. Attendees include plastic surgeons, urologists, 
orthopedic surgeons, and general surgeons; few of these surgeons are comfortable managing polytrauma patients compounded by vascular injury. For these reasons, it is important to assess each individual's skill set and comfort level with these procedures and provide focused training prior to deployment to an austere environment. Several newly introduced civilian courses, such as the Advanced Trauma Operative Management (ATOM) and the Advanced Surgical Skills for Vascular Exposures in Trauma (ASSET) courses, provide excellent focused training on the exposure and management of vascular injuries as well as the techniques for performing extremity fasciotomies. These courses and future platforms using live tissue or realistic simulators will become even more important due to the significant decrease in the exposure of surgical trainees to open vascular surgery.

Patients presenting with severe head injuries, penetrating thoracic, or penetrating abdominal injuries will challenge even the most skilled surgeon, and these patients will drain surgical resources. In the most severe patients, hemorrhage control and transfer may be the best decision rather than attempting a prolonged vascular exposure and repair. If a tourniquet can control extremity hemorrhage, this is perfectly acceptable when the patient is in extremis. In addition, arterial or vein ligation is also an acceptable option for select injuries in the austere setting. The receiving surgeons will reassess all injuries, continue resuscitative measures, and re-evaluate limb salvage as an option.

\section{Conclusions}

The wars in Iraq and Afghanistan have given us the opportunity to evaluate and expand upon vascular management strategies in the austere environment, and to expand the concept of damage control vascular surgery. Keys for the best possible functional outcome include reducing warm ischemia time during transport and limiting the potential for continued injury during the variable period from injury until definitive repair. Early identification, re-establishing flow with temporary shunts, and early or prophylactic fasciotomy should be performed in all injuries when thoracic or truncal injuries demand rapid evacuation or the surgeon is in a austere location with limited resources.

\section{Compliance with Ethical Standards}

Conflict of Interest Drs. Gifford and Arthurs declare no conflicts of interest.

Human and Animal Rights and Informed Consent This article does not contain any studies with human or animal subjects performed by any of the authors.

\section{References}

Papers of particular interest, published recently, have been highlighted as:

- Of importance

1. Rotondo MF, Schwab CW, McGonigal MD, Phillips 3rd GR, Fruchterman TM, Kauder DR, et al. 'Damage control': an approach for improved survival in exsanguinating penetrating abdominal injury. J Trauma. 1993;35(3):375-82. discussion 382-3.

2. Hirshberg A, Mattox KL. Planned reoperation for severe trauma. Ann Surg. 1995;222(1):3-8.

3. Burch JM, Ortiz VB, Richardson RJ, et al. Abbreviated laparotomy and planned reoperation for critically injured patients. Ann Surg. 1992;215(5):476-83. discussion 483-484.

4. Hirshberg A, Wall Jr MJ, Mattox KL. Planned reoperation for trauma: a two year experience with 124 consecutive patients. J Trauma. 1994;37(3):365-9.

5. White JM, Stannard A, Burkhardt GE, Eastridge BJ, Blackbourne LH, Rasmussen TE. The epidemiology of vascular injury in the wars in Iraq and Afghanistan. Ann Surg. 2011;253:1184-9. This article provides an overview of vascular injuries in both Iraq and Afghanistan. IED's have created a new injury pattern that is highlighted in this review.

6. Johansen K, Lynch K, Paun M, Copass M. Non-invasive vascular tests reliably exclude occult arterial trauma in injured extremities. J Trauma. 1991;31(4):515-9. discussion 519-22.

7. Fox CJ, Gillespie DL, Cox ED, Mehta SG, Kragh Jr JF, Salinas J, et al. The effectiveness of a damage control resuscitation strategy for vascular injury in a combat support hospital: results of a case control study. J Trauma. 2008;64(2 Suppl):S99-106. doi:10.1097/TA. $0 \mathrm{~b} 013 \mathrm{e} 3181608 \mathrm{c} 4 \mathrm{a}$. This article provides a critical appraisal of damage control vascular surgery and the impact on mortality rates and limb salvage.

8. Kragh JF, Walters TJ, Baer DG, Fox CJ, Wade CE, Salinas J, et al. Survival with emergency tourniquet use to stop bleeding in major limb trauma. Ann Surg. 2009;249:1-7.

9. Kragh JF, Walters TJ, Baer DG, Fox CJ, Wade CE, Salinas J, et al. Practical use of emergency tourniquets to stop bleeding in major limb trauma. J Trauma. 2008;64:S38-50.

10. Beekley AC, Sebesta JA, Blackbourne LH, Herbert GS, Kauvar DS, Baer DG, et al. Prehospital tourniquet use in operation Iraqi freedom: effect on hemorrhage control and outcomes. J Trauma. 2008;64:S28-37.

11. Stannard A, Morrison JJ, Scott DJ, Ivatury RA, Ross JD, Rasmussen TE. The epidemiology of noncompressible torso hemorrhage in the wars in Iraq and Afghanistan. J Trauma Acute Care Surg. 2013;74(3):830-4.

12. Moore LJ, Brenner M, Kozar RA, Pasley J, Wade CE, Baraniuk MS, et al. Implementation of resuscitative endovascular balloon occlusion of the aorta as an alternative to resuscitative thoracotomy for noncompressible truncal hemorrhage. J Trauma Acute Care Surg. 2015;79(4):523-32.

13. Gifford SM, Eliason JL, Clouse WD, et al. Early versus delayed restoration of flow with a temporary vascular shunt reduces circulating markers of injury in a porcine model. J Trauma. 2009;67(2): 259-65. This article examines temporary arterial shunt placemement in porcine model. This is the foundation by which temporary shunts have become the preferred method for early revascularization in austere settings.

14. Burkhardt GE, Gifford SM, Propper B, Spencer JR, Williams K, Jones $\mathrm{L}$, et al. The impact of ischemic intervals on neuromuscular 
recovery in a porcine (Sus scrofa) survival model of extremity vascular injury. J Vasc Surg. 2011;53(1):165-73.

15. Clouse WD, Rasmussen TE, Peck MA, Eliason JL, Cox MW, Bowser AN, et al. In-theater management of vascular injury: 2 years of the Balad Vascular Registry. J Am Coll Surg. 2007;204: 625-32.

16. Rasmussen TE, Clouse WD, Jenkins DH, Peck MA, Eliason JL, Smith DL. The use of temporary vascular shunts as a damage control adjunct in the management of wartime vascular injury. $\mathrm{J}$ Trauma. 2006;61:8-15.

17. Chambers LW, Freen DJ, Sample K, Gillingham BL, Rhee P, Brown $\mathrm{C}$, et al. Tactical surgical intervention with temporary shunting of peripheral vascular trauma sustained during operation Iraqi freedom: one unit's experience. J Trauma. 2006;61:824-30.

18. Woodward EB, Clouse WD, Eliason JL, et al. Penetrating femoropopliteal injury during modern warfare: experience of the Balad Vascular Registry. J Vasc Surg. 2008;47(6):1259-64. discussion 1264-1265.

19. Taller J, Kamdar JP, Greene JA, Morgan RA, Blankenship CL, Dabrowski $\mathrm{P}$, et al. Temporary vascular shunts as initial treatment of proximal extremity vascular injuries during combat operations: the new standard of care at Echelon II facilities? J Trauma. 2008;65(3):595-603.

20. Rasmussen TE, Clouse WD, Jenkins DH, et al. Echelons of care and the management of wartime vascular injury: a report from the 332nd EMDG/Air Force Theater Hospital, Balad Air Base, Iraq. Perspect Vasc Surg Endovasc Ther. 2006;18(2):91-9.

21. Fox CJ, Gillespie DL, O'Donnell SD, et al. Contemporary management of wartime vascular trauma. J Vasc Surg. 2005;41(4):638-44.

22. Gifford SM, Aidinian G, Clouse WD, Fox CJ, Porras CA, Jones WT, et al. Effect of temporary shunting on extremity vascular injury: an outcome analysis from the global war on terror vascular injury initiative. J Vasc Surg. 2009;50:549-56. This article crtically evaluates the impact of fasciotomies for limb salvage operations.

23. Ritenour AE, Dorlac WC, Fang R, Woods T, Jenkins DH, Flaherty $\mathrm{SF}$, et al. Complications after fasciotomy revision and delayed compartment release in combat patients. J Trauma. 2008;64(2 Suppl): S153-61. discussion S161-2. 